\title{
Improved Reconstruction of Global Precipitation since 1900
}

\author{
THOMAS M. SMITH \\ NOAA/NESDIS/STAR, and ESSIC/CICS, University of Maryland, College Park, College Park, Maryland \\ PHILLIP A. ARKIN AND Li REN \\ ESSIC/CICS, University of Maryland, College Park, College Park, Maryland
}

SAMUEL S. P. SHEN

San Diego State University, San Diego, California

(Manuscript received 15 December 2011, in final form 4 May 2012)

\begin{abstract}
An improved land-ocean global monthly precipitation anomaly reconstruction is developed for the period beginning in 1900. Reconstructions use the available historical data and statistics developed from the modern satellite-sampled period to analyze variations over the historical presatellite period. This paper documents the latest in a series of precipitation reconstructions developed by the authors. Although the reconstruction principle is still the minimization of mean-squared error, this latest reconstruction includes the following three major improvements over previous reconstructions: (i) an improved method that first produces an annual first guess, which is then adjusted using a monthly increment analysis; (ii) improved use of oceanic observations in the annual first guess using a canonical correlation analysis; and (iii) reinjection of gauge data where those data are available. These improvements allow more confident analyses and evaluations of global precipitation variations over the reconstruction period. Quantitative error estimates for the reconstruction are being developed and will be documented in a later paper.
\end{abstract}

\section{Introduction}

Surface temperature and precipitation are arguably the most basic climatic parameters. Precipitation variations control freshwater availability, food production, disease outbreaks, floods, and droughts. Models suggest that precipitation will change with global temperature, and monitoring is critical for assessing changes both globally and regionally.

Although many land regions have long precipitation records from gauges, there are spatial gaps in the sampling for undeveloped regions, areas with low populations, and over oceans. Since 1979 satellite data have been used to fill in those sampling gaps. Over longer periods gaps can only be filled using reconstructions or

Corresponding author address: Tom Smith, NOAA/NESDIS/ SCSB, and ESSIC/CICS, University of Maryland, College Park, 5825 University Research CT, Suite 4001, College Park, MD 20740.

E-mail: tom.smith@noaa.gov reanalyses. Here a statistical analysis is used to produce a filled field of data from relatively sparse observations. We call this type of analysis a reconstruction. We use the word reanalysis to denote a dynamic analysis using data assimilation (e.g., Compo et al. 2006).

Precipitation variations over oceans and land regions with few data are important because they help to show large-scale variations in precipitation over time. Although individual precipitation events may be chaotic, the mean large-scale precipitation shows high- and lowprecipitation zones. Important large-scale precipitation features can be seen in maps of the 1979-2008 average Global Precipitation Climatology Project (GPCP; Huffman et al. 1997a; Adler et al. 2003; Huffman et al. 2009) for averages over December-February (DJF), June-August (JJA), and all months (Fig. 1). An important high-precipitation zone is the intertropical convergence zone (ITCZ), where strong convective precipitation tends to develop, typically a few degrees north of the equator. At roughly $30^{\circ}$ latitude there are zones of atmospheric subsidence with low precipitation, where many of 


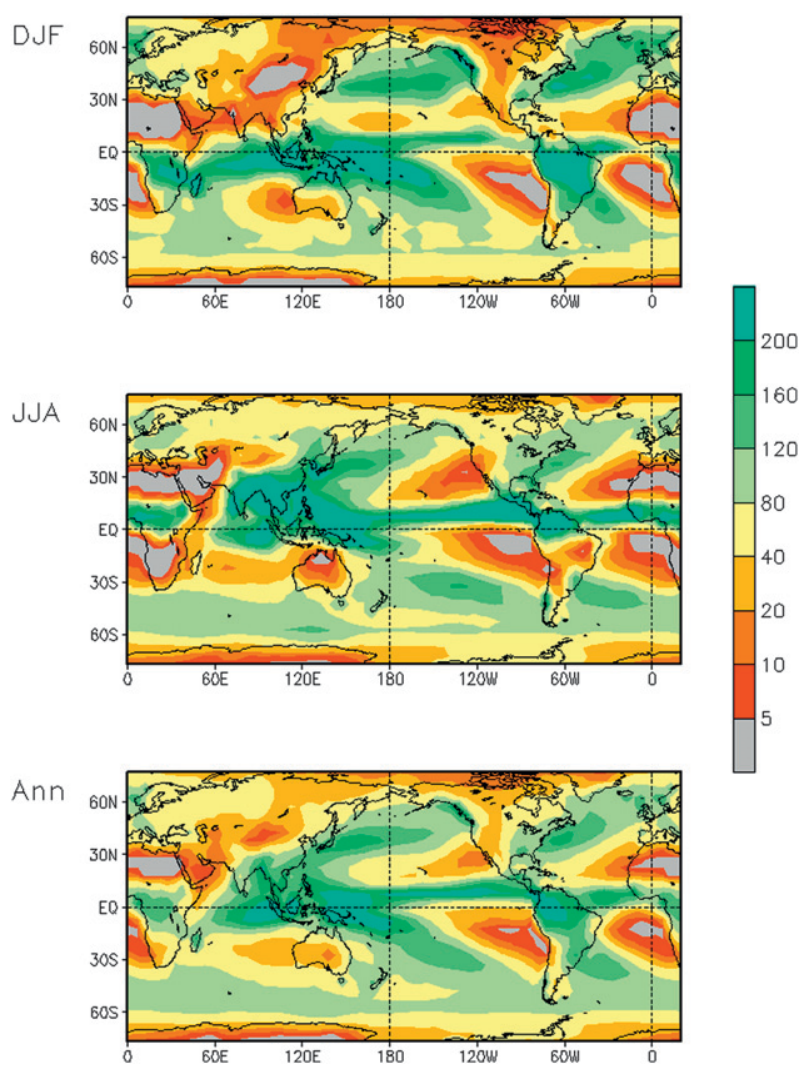

FIG. 1. GPCP 1979-2008 seasonal DJF, JJA, and annual climatology.

the earth's deserts tend to form. Poleward of that are the midlatitude storm tracks. Large seasonal changes occur in the tropics resulting from the monsoons and seasonal shifts of the ITCZ. In the extratropics the magnitudes are smaller but the seasonal variations are important to the supply of freshwater in those regions. Shifts, expansions, or intensifications of these zones can have a large influence on precipitation over land regions and thus can influence the spatial-temporal availability of freshwater. Large-scale complete data that include oceanic regions are necessary for detecting and monitoring possible changes in the large-scale precipitation patterns. Dynamic models are useful tools to help identify physical processes that can cause precipitation changes, but observation-based analyses are needed for model validation.

Statistical reconstructions require dense modern-period base data for generating statistics. Satellite-based analyses are typically used to generate spatially complete statistics. Several different satellite and gauge analyses are available based on different analysis methods. The most advanced of these analyses rely on the improved precipitation estimates derived from passive microwave observations, available with enough density for high-frequency analyses since about 1998. Analyses such as Climate Prediction Center (CPC) morphing method (CMORPH; Joyce et al. 2004), Tropical Rainfall Measuring Mission (TRMM) Multisatellite Precipitation Analysis (TMPA; Huffman et al. 1997b), and Precipitation Estimation from Remotely Sensed Information using Artificial Neural Networks (PERSIANN; Hsu et al. 1997; Sorooshian et al. 2000) all use high space-time resolution infrared (IR) observations in combination with instantaneous estimates derived from passive microwave observations to derive precipitation on time scales of 1-3 $\mathrm{h}$ and spatial scales of $0.25^{\circ}$ for the tropics and midlatitudes. These analyses are becoming widely used for monitoring and understanding precipitation variability on fine scales, but their length of record is too brief to be used to compute statistics for a century-long statistical reconstruction.

Another satellite analysis approach is to use a wide range of different infrared and microwave satellite estimates of precipitation merged with gauge estimates. Two principal datasets using this approach have been published: the previously mentioned Global Precipitation Climatology Project (GPCP) and the CPC Merged Analysis of Precipitation (CMAP; Xie and Arkin 1996, 1997). In these datasets, time-averaged satellite-derived estimates are combined with an analysis based on gauge observations beginning in 1979 to yield monthly global analyses at $2.5^{\circ}$ spatial grid. CMAP uses a linear combination of the available satellite-derived estimates, while in GPCP different satellite estimates are adjusted relative to each other to minimize satellite-to-satellite biases before they are merged. The available blend of satellitederived estimates provides good spatial coverage, but neither the infrared nor the microwave estimates can be used over snow and ice surfaces. For GPCP some highlatitude regions are filled using lower-quality estimates based on Television and Infrared Observation Satellite (TIROS) Operational Vertical Sounder (TOVS) satellite estimates of deep extensive clouds, which are used to infer precipitation.

The merged satellite estimate is blended with gauge estimates in both products. CMAP additionally adjusts tropical and midlatitude oceanic values to match gauge observations in the central and western equatorial $\mathrm{Pa}$ cific Ocean. We have found that using GPCP base data yields a more realistic reconstruction of twentieth-century precipitation (Smith et al. 2008b, 2009a), although the specific reasons are not completely clear. Although a number of satellite estimates are merged for the GPCP, the intersatellite bias adjustments remove the most severe relative biases between satellites. In addition, recent salinity budget studies suggest that GPCP precipitation provides a better salinity balance over the oceans compared to other satellite-based analyses (Yu 2011; Ren and 
Riser 2009; Yaremchuk et al. 2005; Yaremchuk 2006). Here the GPCP-merged satellite and gauge analysis is used as the base data to form statistics for the reconstruction of historical precipitation. Because of compromises made in forming GPCP these base data can introduce some uncertainty in the reconstructions, particularly at high latitudes, further improvements in such global analyses are desirable.

Several precipitation reconstructions have been developed, with varying degrees of success. Xie et al. (2001) and Efthymiadis et al. (2005) each developed reconstructions that give useful skill in the tropics, although their reconstructions have lower extratropical skill. A series of reconstructions were developed by Smith et al. (2008b, 2009a, 2010) to improve the overall skill of historical reconstructions. Those studies, summarized in section $2 b$, showed the potential for skillful oceanic precipitation reconstruction beginning in 1900. Each study provides some improvement. Because the historical precipitation data used for reconstruction over ocean areas are sparse, we continue to seek improvements in analysis methods, understanding problems, and uncertainty quantification. The reconstruction described here is the latest in the series of studies. It gives the greatest improvement in the spatial resolution of oceanic precipitation patterns, along with some improvements in land-area precipitation.

The greatest improvement comes from development of a first-guess annual anomaly analysis that includes oceanic data. The first guess is a global analysis using a limited number of spatial reconstruction modes. Those annual modes are used to filter a combination of annual gauge data and oceanic estimates made using a canonical correlation analysis (CCA). The CCA annual anomaly estimates are based on annual sea surface temperature (SST) and sea level pressure (SLP) anomalies. Combining the CCA estimates with gauges and filtering them using the spatial modes allows us to retain most of the signal that they resolve while adjusting for spatial biases that appear to be present in the CCA. The annual first guess is adjusted using a monthly increment reconstruction based on a larger number of spatial modes. Historical gauge-based increments are used with those modes to produce the increment analysis, which is added to the first-guess analysis. Finally, the monthly gauge data are statistically reinjected into the analysis where those data are available. In Smith et al. (2010), the CCA data are also used over oceanic regions, but in that reconstruction the CCA data are not combined with gauges and filtered using global modes. The presence of apparent spatial biases over oceanic regions in the Smith et al. (2010) analysis motivated the development of this improved reconstruction. In addition, the reinjection of gauge data over land regions was developed since that study.

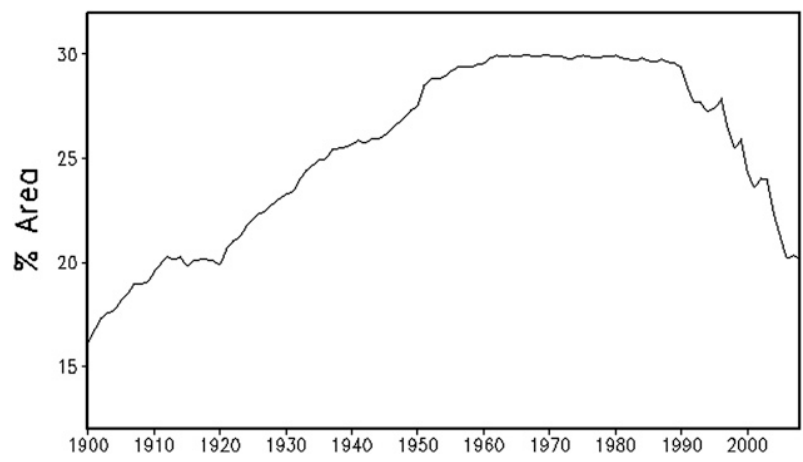

FIG. 2. Annual average percent of $5^{\circ}$ areas sampled in the GHCN gauge analysis.

\section{Historical data and precipitation reconstructions}

The historical data used for the improved reconstruction are the same as those used in our earlier reconstructions, and are briefly described here. The earlier reconstructions by Smith et al. (2008b, 2009a, 2010) are described to illustrate how those data may be used, and to discuss the strengths and weaknesses in the earlier reconstructions.

\section{a. Historical data}

Gauge-based analyses provide the most direct measurement of historical precipitation. In reconstructions the gauge analyses may be used to anchor large-scale precipitation for analysis over undersampled areas. Our earlier studies used several historical gauge analyses. One is the Global Historical Climatology Network (GHCN; Vose et al. 1998), produced by the National Climatic Data Center. The gridded GHCN is a monthly dataset on a $5^{\circ}$ spatial grid, 1900-2008. Other gaugebased gridded datasets were evaluated, including the Global Precipitation Climatology Center (GPCC), version 4 (Schneider et al. 2008; Rudolf 2005) and the University of East Anglia Climate Research Unit (CRU) analysis (Hulme et al. 1998). Both of these datasets are available on a $2.5^{\circ}$ monthly grid, and are averaged to the GHCN $5^{\circ}$ monthly grid. The GPCC dataset begins in 1901 while the CRU begins in 1900. Both yield reconstructions that are qualitatively similar to GHCN-based reconstructions. However, the GHCN has a slightly longer period and uses less gap filling, and therefore was used for this study.

The GHCN analysis of gauge data has the best sampling beginning about 1950 , when most $5^{\circ}$ regions over land have enough data for an analysis value (Fig. 2). From 1900 to about 1950 the sampling area gradually increased as more global stations became available. After about 1990 sampling begins to gradually decrease, resulting from time 
lags in incorporating some data and also some stations being discontinued.

Both SST and SLP historical analyses have been used to reconstruct annual historical precipitation anomalies. The SSTs are from the analysis of Smith et al. (2008a) and the SLPs are from the analysis of Allan and Ansell (2006). As discussed in Smith et al. (2009a), anomalies of annual SST and SLP have been found to correlate with large-scale annual precipitation anomalies, and therefore they can be used as proxies for historical precipitation. Individual precipitation events have small time and space scales, and in any case precipitation is typically not measured by ships. In contrast, the available monthly SST and SLP tend to have larger scales and have been measured by ships since before 1900 (Woodruff et al. 1998). Although ship measurements have some spatial biases, they are sufficient for analyses of both historical fields. The existence of the SST and SLP makes it possible to measure their correlations with mean precipitation for large spatial and temporal scales. Evaluations suggest that the SST and SLP correlations may not be useful for anomaly reconstruction on temporal scales shorter than annual. Monthly correlations were evaluated, but the results were poor compared to the annual correlations. On longer time scales the background SST and SLP appear to be more directly linked to precipitation, while on shorter time scales dynamics not as closely linked to those background conditions may exert more influence.

\section{b. Recent historical reconstructions}

Building on findings from earlier reconstructions of SST and precipitation, Smith et al. (2008b) developed a direct reconstruction of monthly precipitation. That reconstruction used spatial covariance empirical orthogonal functions (EOFs) for the following three separate areas: $80^{\circ}-20^{\circ} \mathrm{S}, 30^{\circ} \mathrm{S}-30^{\circ} \mathrm{N}$, and $20^{\circ}-80^{\circ} \mathrm{N}$. That separation was done to keep the much larger tropical variability from dominating extratropical variations. The reconstruction was produced by fitting the available monthly gauge anomalies to the set of EOF modes in each region using a least squares regression procedure. In each region the reconstruction is the weighted sum of the modes. For that earlier reconstruction the three regions are merged by interpolation as a function of latitude in their overlap regions.

That direct reconstruction of monthly anomalies gave physically reasonable monthly to interannual variations over the oceans. In the overlap regions where the analyses were merged the variance was damped, because the linear interpolation smoothed the overlap region. The greatest potential problem with that reconstruction was its multidecadal variations, which were inconsistent with the theoretical understanding of how large-scale multidecadal precipitation should vary. Both theory and climate models suggest that precipitation should increase with warming temperatures, because warmer air temperatures had a much larger saturation vapor pressure. Therefore, both global evaporation and precipitation are expected to increase under warming conditions (Held and Soden 2006; Allan and Soden 2008). Over land the global average of gauge data indicates a positive trend over the twentieth century (Trenberth et al. 2007). Beginning in about 1950 the ocean-area multidecadal variations in the Smith et al. (2008a) reconstruction were consistent with the theory. Before 1950, when sampling was less frequent and the reconstruction was less reliable, the variations were inconsistent with the theory. That inconsistency in a period with lower sampling made us suspicious of the multidecadal signal before 1950 .

Historical precipitation data are extremely limited over oceans. There are coastal and island gauge observations, but there are also large expanses of oceans far from any gauges. Present weather is recorded in ship logs, but it is difficult to convert that to large-scale precipitation, both because such records do not give precipitation amounts and because of the short space and time scales of individual precipitation events. Therefore, to reconstruct historical precipitation on large scales we used correlations between annual precipitation anomalies and annual SST and SLP anomalies. We did this by using a CCA. Although both SST and SLP have sampling errors, they are both sampled much better than oceanic precipitation, and therefore may be used to infer some aspects of precipitation variations that may not be detectable from historical precipitation data alone. The CCA method is described in detail by Barnett and Preisendorfer (1987), and its application to historical precipitation reconstruction is described by Smith et al. (2009a). Briefly, the CCA correlates fields of predictors with predictand fields. It is similar to ordinary regression, except that ordinary regression is between local points on the fields, while CCA can relate regions of one field with different regions on another. The CCA used the combined annual SST and SLP anomalies as predictors, and the annual precipitation anomalies as predictands. The CCA was trained using GPCP data over 1979-2004. The training period did not extend past 2004 because the SLP data for the later years were from updates that had different a variance than the SLP analysis in earlier years, and we did not want that change in variance to influence the correlations.

The CCA relationships developed in the training period were used with the historical annual SST and SLP anomalies to reconstruct the annual precipitation anomalies globally. Examination of the global averages over 
land showed that the CCA reproduced the major gaugeaveraged variations over the historical period, before the training period of 1979-2004, as shown by Smith et al. (2009a). Because the gauges were not used in the $\mathrm{CCA}$ reconstruction that result was encouraging. In the modern GPCP period, the global averages over oceans were reproduced by the CCA, although that was a comparison of dependent data. Over the historical period, the CCA indicated increasing precipitation with warming, consistent with theory and models. We believe that its consistency with theory, in combination with the other comparisons discussed, makes the CCA a better analysis of multidecadal ocean-area variations, compared to the direct monthly reconstruction that had preceded it.

Although the CCA is in some ways an improvement for analysis of multidecadal variations, it also has some weaknesses. Examination of the trends over the GPCP period showed that the spatial scales of the CCA trends are several times larger than the spatial scales of the GPCP trends. The overall location of the positive and negative trends in both are similar, but the CCA trend patterns are more spread out compared to GPCP. Those large spatial scales of trends are maintained over the entire CCA reconstruction. We found that the likely cause for the large spatial scales of trends was that the correlated data used for the CCA simply could not give better spatial resolution. Tests were performed using local pseudoprecipitation constructed from ordinary local regression of SST anomalies against precipitation anomalies. That pseudoprecipitation was used for reconstruction, and yielded trend patterns with spatial scales and magnitudes similar to those from the CCA. Another test reconstruction used joint EOFs of normalized annual precipitation and SST to develop joint SST and precipitation modes. Those joint modes were then used for reconstruction using the historical normalized SST to find weights for the joint modes. Again the trend scales and magnitudes resembled those of the CCA. Although these test results were in many ways similar to CCA results, their skill was less when tested against dependent data and in cross-validation tests, so we only use them for evaluating the CCA.

To use the strengths of both the direct monthly reconstruction of Smith et al. (2008b) and the indirect annual reconstruction of Smith et al. (2009a), we developed the merged reconstruction of Smith et al. (2010). The merged reconstruction uses the multidecadal variations of Smith et al. (2009a) over the oceans. Multidecadal signals are separated from higher-frequency signals using a 7-yr filter. The annual averages of the direct reconstruction and the CCA were filtered and the direct reconstruction multidecadal signal was replaced with the CCA multidecadal signal over ocean areas. The oceanic higher-frequency signals were not changed. In addition, over land variations where gauges are available the direct signal was not changed. This merged analysis should yield better multidecadal ocean-area variations compared to Smith et al. (2008b), while retaining the strengths of that reconstruction. It will, however, still retain the CCA problems discussed above.

\section{EOF-based reconstructions}

The improved reconstruction method is described in this section. This includes the comparison of results using only gauge data and gauge data supplemented by oceanic CCA results to show the influence of the additional data. The EOF method described here is different from similar methods used in earlier studies for two reasons. One is that here we develop an annual firstguess analysis using one set of modes and then adjust that using a monthly increment analysis using a second set of modes. The other is that both annual and monthly increment modes used here are computed globally and not separately for different regions, as was done earlier. The CCA used here is the analysis described by Smith et al. (2009a). The difference here is how results of that CCA analysis are used. We test the method with and without the CCA results to show its influence on the improved analysis.

\section{a. Method}

The basic EOF reconstruction method was described in Smith et al. (1996, 1998). Here we summarize the method and describe how it is used for the improved analysis. The basic idea is to use a set of covariance EOFs to estimate historical variations by estimating time series for the EOFs using the incomplete historical data. Because the data are incomplete, the historical time series values are estimated by minimizing the mean-squareerror (MSE) of the fit compared to the available data. A screening procedure is used to remove EOF modes that are not adequately sampled by the historical data.

The reconstruction as a function of space and time is a weighted linear combination of a set of $M$ EOF modes,

$$
F(x, t)=\sum_{n=1}^{M} w_{n}(t) E_{n}(x) .
$$

Here the weight $w_{n}(t)$ is the time series value for time $t$ and mode $n$, and the EOF mode $E_{n}(x)$ is a function of only spatial location $x$. We optimize the weights by minimizing the integrated mean square differences (MSD) between the reconstruction $F(x, t)$ and data $D(x, t)$ over the data-sampled domain, 


$$
\varepsilon^{2}=\sum_{x}[D(x, t)-F(x, t)]^{2} \delta(x, t) a(x)
$$

Here the delta function defines data sampling, with $\delta(x, t)=1$ if there is sampling for the spatial location and time, and $\delta(x, t)=0$ if there is no sampling. The function $a(x)$ denotes the relative area of each location, which can be estimated by the cosine of the latitude for the grid box's centroid. To minimize the MSD, we differentiate $\varepsilon^{2}$ with respect to weights $w_{n}$ for $n=1,2, \ldots, M$, and obtain the set of linear equations for the optimal weights,

$$
\begin{aligned}
& \sum_{n=1}^{M} w_{n} \sum_{x} E_{n}(x) E_{k}(x) \delta(x) a(x) \\
& \quad=\sum_{x} D(x, t) E_{k}(x) \delta(x) a(x), \text { for } k=1, \ldots, M .
\end{aligned}
$$

This system of equations is solved for each time step to give a set of weights for that time step for each mode. The set of spatial modes is assumed to be stationary, and therefore historical variations are assumed to be linear combinations of the modes. Thus, all reconstructed variations are a weighted linear sum of the basis modes. The relative variance associated with each mode may change in time because the time series is computed separately for each time. That means that climate processes dominant in the base period may not be dominant in the historical period.

Note that with complete sampling orthonormal property of the EOFs yields

$$
\sum_{x} E_{n}(x) E_{k}(x) a(x)=\delta_{n k},
$$

which is 1 if $n=k$ and 0 otherwise. Thus, with complete sampling Eq. (2) reduces to

$$
w_{n}=\sum_{x} D(x, t) E_{n}(x) a(x) .
$$

This is the orthogonal projection of the completely sampled data onto the mode $E_{n}(x)$. Because historical sampling is incomplete, it is necessary to use Eq. (2) to find the best-fit weights over the historical period.

A problem can occur with this type of reconstruction when data become too sparse to sample a mode. All historical data will contain noise in addition to signal. When there are many observations random noise will be filtered out because it will not fit the spatial covariance patterns. However, if there are too few data, then noise can cause large inflation in one or more of the modes, which will spread the error over the mode's spatial domain. To avoid that problem we test the sampling of each mode and screen out modes that are not adequately sampled. The reconstruction is then performed using only those modes that pass the screening test. The screening test was described by Smith et al. (1998) and is summarized here.

To screen modes we measure the fraction of the variance for each mode sampled by the available data at each time. At each point the variance of a mode is proportional to the mode squared. Thus, the fraction of variance sampled by each mode can be defined as

$$
f_{n}=\frac{\sum_{x} E_{n}^{2}(x) \delta(x) a(x)}{\sum_{x} E_{n}^{2}(x) a(x)} .
$$

Using properly scaled orthogonal modes the denominator in Eq. (4) is equal to 1 . When this fraction of sampling gets below a critical value for a mode, that mode is screened out of the reconstruction. Cross-validation testing has shown that the critical fraction for screening is usually $0.05-0.10$, although there is usually little difference in cross-validation error using values between 0.10 and 0.20 . When the critical value is set too high error increases due to the exclusion of well-supported modes.

The improved reconstruction is formed using the basic method described above. However, rather than direct reconstruction of monthly anomalies a two-step approach is used using two sets of EOF modes. The initial step is reconstruction of annual average anomalies globally using a limited number of modes to focus on longerperiod variations. The annual GPCP anomalies are used to compute a set of 20 global covariance EOFs, which is about as many annual modes as can be computed from the 1979-2008 record. Annual historical data anomalies are used to reconstruct the annual global anomalies using the method described above. Note that this annual reconstruction is separate from the CCA and could be produced with or without CCA inputs, as discussed below. Because month-to-month variations and noise are filtered out of this analysis, the reconstruction modes and data can focus on multidecadal variations more accurately. Month-to-month variations in the modes would require many more modes in a direct reconstruction, and month-to-month noise could contaminate the weaker multidecadal signal. This annual reconstruction provides a first-guess analysis that can be adjusted to give monthly anomalies.

The annual global reconstruction is adjusted using reconstructed monthly increments. Increments are defined as the difference between the monthly anomaly and the annual anomaly. To avoid possible steps between years the annual anomaly linearly interpolated in time 


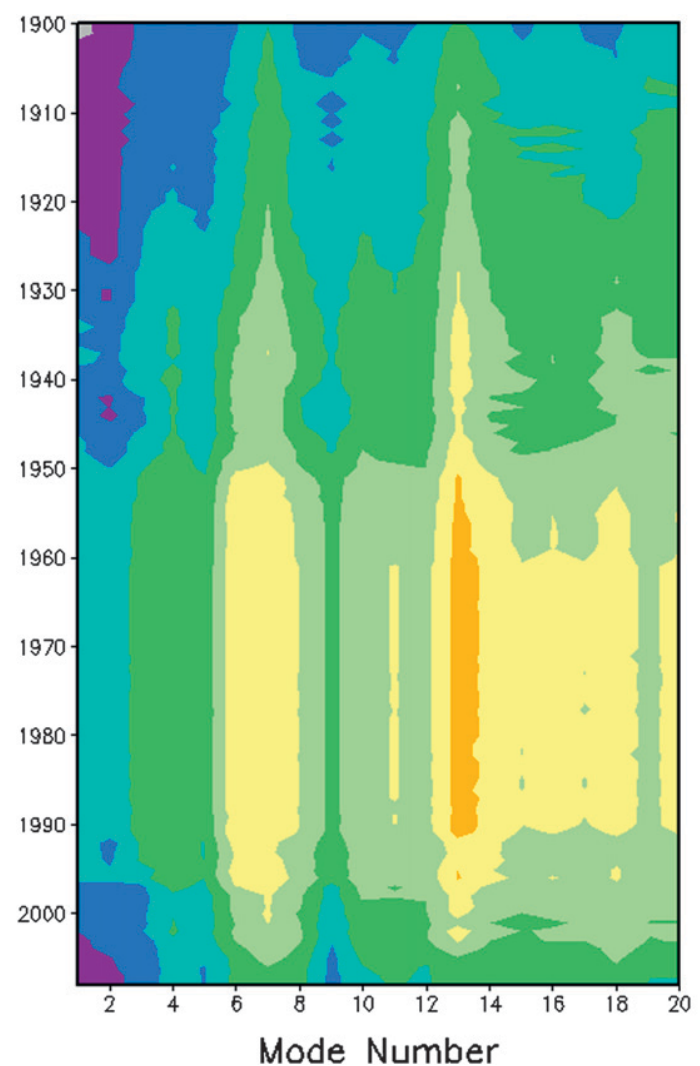

FIG. 3. Percent of variance sampled for each of the 20 global annual reconstruction modes using annual GHCN.

to the month of the year. This correction of the annual anomaly is what needs to be analyzed to adjust the annual first guess. The GPCP increments are used to compute a set of 40 monthly global increment EOFs. Historical data increments are defined as differences between the data and the global annual reconstructed anomaly linearly interpolated to the month. Those data increments are used to compute the monthly global increment reconstruction. Adding the increment to the annual reconstruction gives the reconstructed monthly anomaly.

All of these reconstructions are computed on the same $5^{\circ}$ spatial grid as our earlier reconstructions. For both the annual and monthly increment reconstructions, we screen out modes with less than $5 \%$ variance sampling. This relatively low sampling is assigned so that most modes will be used in the analysis most of the time. For the annual global component of the analysis there are only a few years when sampling falls below $5 \%$ for the first mode (Fig. 3). For the monthly increments we always use the historical GHCN gauge analysis to compute historical weights for the EOFs. For the annual global reconstruction we tested two sets of historical inputs. One is the GHCN gauge analysis alone. The other is the GHCN gauge analysis supplemented using

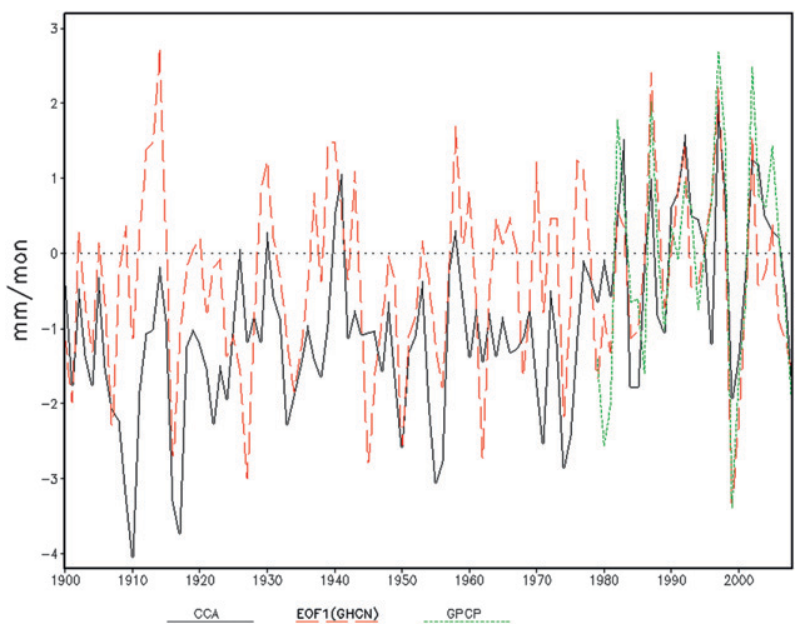

FIG. 4. Annual and ocean-area average precipitation anomalies $\left(75^{\circ} \mathrm{S}-75^{\circ} \mathrm{N}\right)$ of the CCA, the EOF1 using only gauges, and the GPCP satellite-based analysis.

the CCA precipitation estimates in ocean grids for which there is no GHCN estimate. All historical anomaly data are centered onto the same base period before computing the reconstruction. Both reconstructions, with and without CCA, are described and evaluated in later subsections.

\section{b. Reconstruction using gauge data}

The reconstruction methods described above were first tested using historical GHCN gauge data, referred to here as EOF1(GHCN). The annual and ocean averages over $75^{\circ} \mathrm{S}-75^{\circ} \mathrm{N}$ are used for overall comparisons with CCA and GPCP (Fig. 4). In the satellite period both reconstructions compare well to GPCP. That is not surprising because GPCP is used to form reconstruction statistics for both. The satellite period comparisons do show the potential for using relatively sparse gauge data for reconstruction globally.

Over the full reconstruction period both CCA and EOF1(GHCN) indicate similar interannual variations, although interannual variations in $\mathrm{EOF} 1(\mathrm{GHCN})$ tend to be stronger than those from CCA. The CCA and EOF1(GHCN) have qualitatively consistent multidecadal variations over the full period, although the CCA multidecadal change is stronger than that indicated by EOF1 $(\mathrm{GHCN})$. The CCA linear trend over the full period is $1.6 \mathrm{~mm}$ month $^{-1}(100 \mathrm{yr})^{-1}$, while for EOF1(GHCN) it is $0.3 \mathrm{~mm}$ month $^{-1}(100 \mathrm{yr})^{-1}$. After about 1980 the two have similar multidecadal tendencies, but the CCA has more multidecadal change before then.

The CCA was developed to use ocean information in order to have an improved estimate of multidecadal ocean-area variations. Because the CCA is strongly influenced by SST it is sensitive to SST changes, which is 


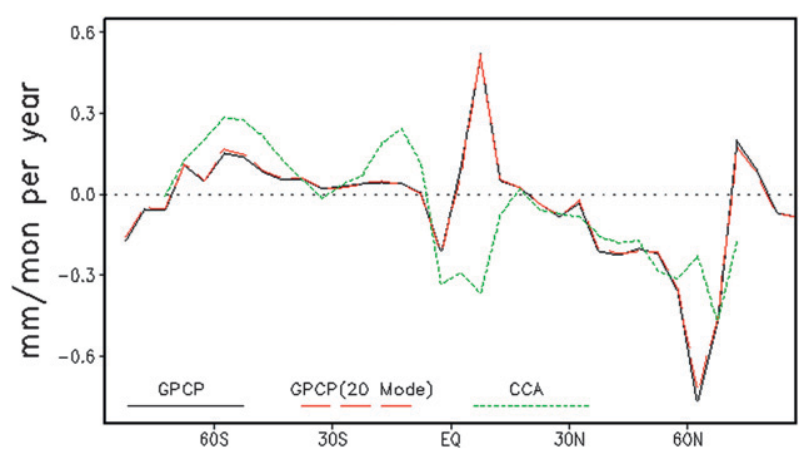

FIG. 5. Ocean-area zonal average trends over 1979-2008 from the annual GPCP, the annual GPCP filtered using the 20 annualglobal reconstruction modes, and the CCA.

especially apparent in the 1970 s when there was a climate shift in SST (Trenberth and Hurrell 1994). Comparisons of an ensemble of coupled model output showed that the average ocean CCA multidecadal signal is $2-3$ times larger than the coupled model ensemble multidecadal signal (Smith et al. 2009b). Recent analyses of salinity for the second half of the twentieth century supports the larger CCA signal (Durack et al. 2012). Without oceanic data the gauge-only reconstruction is not able to fully resolve the stronger low-frequency variations apparent in the CCA.

Compared to the earlier period, since about 1950 the EOF1(GHCN) averages indicate a trend that is more similar to that from the CCA. Before 1950 is the time when GHCN sampling is lowest (Figs. 2 and 3), suggesting that the greater damping in that period may be due to inadequate resolution of some of the modes.

The 20 reconstruction modes used for the annual component of the EOF1(GHCN) reconstruction are capable of representing multidecadal variations if those modes are resolved by the data. This is shown by comparing trends in the annual GPCP with trends from annual GPCP filtered using the 20 modes (Fig. 5). Practically all of the zonal trend over the satellite period can be described using the set of reconstruction modes. The problem is being able to adequately sample enough modes through the entire reconstruction period. For comparison the zonal CCA trend over the same period is also shown. On very large scales the zonal CCA trend resembles the GPCP trend, with a generally decreasing trend from south to north. However, the CCA fails to resolve the narrow positive trend just north of the equator. Examination of maps of trends shows that the CCA trends have similar shapes, but they are spatially spread over large regions with some shifts in latitude. In contrast to that the GPCP trends tend to have smaller spatial scales. These zonal average trends suggest that compared to the CCA, the EOF1 could yield better spatial resolution of multidecadal variations in periods when its modes can be adequately resolved.

Although most global annual modes are used in the annual component of the EOF1(GHCN) reconstruction, the sparser sampling early in the analysis period may produce larger errors in the weights of some of the modes used in that period, which could influence the multidecadal signal. To test the influence of sampling we reconstructed the base GPCP data using sampling masks to match the GHCN sampling for 1900 and 1950. Results were compared to the reconstruction with the full GPCP sampling. Average ocean-area trends were evaluated. This test showed that the trend with 1950 GHCN sampling differed from the full GPCP trend by about $10 \%$, while the trend with 1900 sampling differed by about $200 \%$. The test shows that nearly full land sampling similar to the sampling available in 1950 may be sufficient to resolve most ocean-area multidecadal variations. The much worse match using 1900 sampling shows clearly that multidecadal variations from the early twentieth century are less reliable and more historical precipitation information is needed to anchor the analysis for the period before 1950 . The next subsection describes a way to reconstruct using more historical information on multidecadal oceanic precipitation.

\section{c. Reconstruction using both CCA and gauge data}

As described above, the EOF1 modes for the annual component of the reconstruction can resolve most multidecadal variations if the historical sampling is adequate. The annual GHCN gauge analysis resolves most of the variance associated with those modes since 1950, but it resolves much less in earlier years. The CCA uses ocean data to reconstruct ocean-area annual variations. For very large spatial scales, roughly $30^{\circ}$ or larger, CCA multidecadal variations are consistent with GPCP in the overlap period, suggesting that the CCA may be superior to the EOF1(GHCN) on such large scales because of its use of oceanic data. The problem with the CCA is that it does not resolve multidecadal variations on finer spatial scales.

To incorporate ocean data into the EOF1 reconstruction, the ocean-area CCA precipitation anomalies are used as additional input in the global annual EOF1component of the reconstruction. The GHCN annual anomalies are still used where they are available, and CCA annual anomalies are only used for ocean grid squares that do not contain a GHCN estimate. This annual global component of the EOF1 reconstruction has good sampling for all 20 modes in all years. Because the CCA is an annual analysis used here to improve multidecadal variations, the monthly increment adjustments to this analysis are computed using only the 


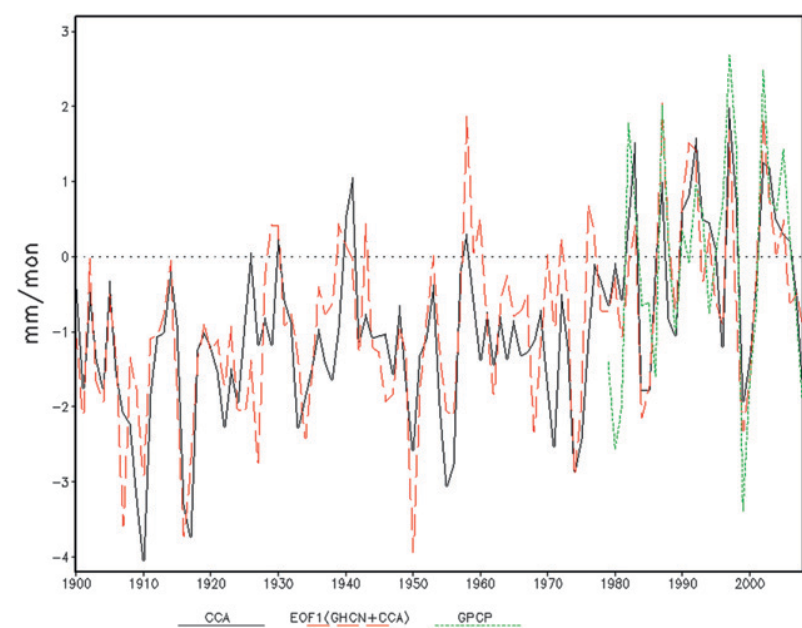

FIG. 6. Annual and ocean-area average precipitation anomalies $\left(75^{\circ} \mathrm{S}-75^{\circ} \mathrm{N}\right)$ of the CCA, the EOF1 incorporating both CCA and gauges, and the GPCP satellite-based analysis.

increment GHCN gauge analyses. We call this analysis $\mathrm{EOF} 1(\mathrm{GHCN}+\mathrm{CCA})$.

Incorporation of both CCA and GHCN data yields EOF1 near-global averages with roughly the same multidecadal variations as the CCA over ocean areas (Fig. 6). For EOF1(GHCN + CCA) the ocean average trend over the analysis period is $1.5 \mathrm{~mm}$ month $^{-1}(100 \mathrm{yr})^{-1}$, compared to 0.3 for EOF1(GHCN) and 1.6 for the CCA. However, the annual EOF1(GHCN + CCA) has larger interannual variations than the CCA.

Ocean-area zonal average trends over the entire analysis period show how the inclusion of CCA influences multidecadal variations (Fig. 7). The CCA has a large, broad positive trend centered on the equator. For the EOF1(GHCN) the tropical maximum is weaker, narrower, and shifted to north of the equator. Incorporation of both GHCN and CCA in the EOF1 keeps the location and scale of the tropical maximum, but increases its strength. It also removes a negative trend in EOF1 (GHCN) centered just south of the equator, replacing it with a modest positive trend. By filtering the CCA and annual GHCN using the global annual modes we are able to keep the oceanic information provided by the CCA but with finer resolution of spatial scales in the multidecadal variations. This analysis resolves multidecadal variations that cannot be resolved using historical GHCN alone.

\section{d. Reinjection of gauge data}

We use an optimum interpolation (OI) analysis to adjust the reconstruction using the GHCN gauge data. The $\mathrm{OI}$ analysis is done each month on $\mathrm{GHCN} 5^{\circ}$ gauge $(G)$ minus reconstruction $(R)$ differences. Both the reconstruction and gauge data are anomalies, recentered

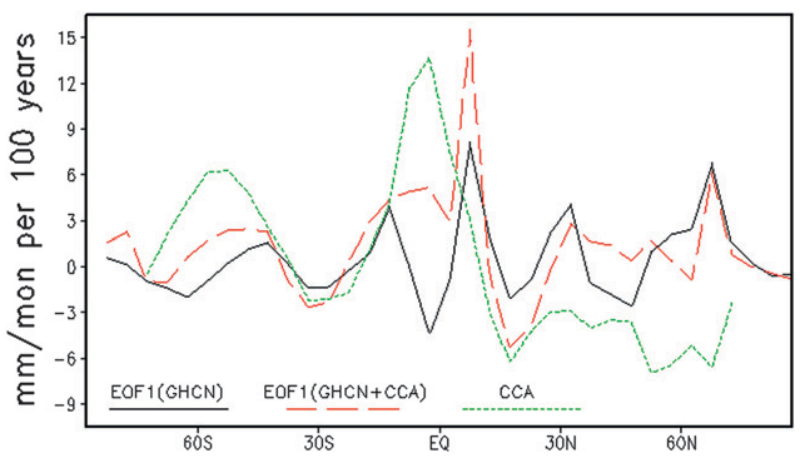

FIG. 7. Ocean-area zonal average trends over 1900-2008 from EOF1(GHCN), EOF1(GHCN + CCA $)$, and CCA.

for this step so that their 1900-99 mean is zero. Because the gauge data can be noisy, the differences are checked before analysis and large differences are not used for reinjection. The root-mean-square difference (RMSD) between gauges and reconstructions across latitude bands are computed. Because of sparse matchups in some regions, the zonal RMSD is smoothed by averaging over three latitude bands, or $15^{\circ}$ of latitude. The individual absolute differences are then compared to the smoothed zonal RMSD. If the individual absolute value is greater than 20 times, the RMSD it is not used. For the EOF1 reconstruction this eliminated an average of just over two differences per year.

The OI uses isotropic spatial scales set to one equatorial gridbox distance, $5^{\circ}$ or $555.95 \mathrm{~km}$. This is about the smallest spatial scale possible for data on this spatial grid and should preserve the greatest spatial resolution possible with this grid. The noise-to-signal variance ratio is set to 0.5 in midlatitudes $\left(25^{\circ}-60^{\circ}\right)$ and 1.0 poleward of $65^{\circ}$, with a linear interpolation between $60^{\circ}$ and $65^{\circ}$ latitude. The high-latitude values can be noisier due to problems associated with blowing snow. Such highlatitude gauges are sparse and do not greatly influence the analysis except where they are locally available, so we use this simple boosting of the noise at high latitudes with no seasonal component for the noise-to-signal ratio. Within $20^{\circ} \mathrm{S}-20^{\circ} \mathrm{N}$ the ratio is 0.5 before 1960 , increasing to 1.0 after 1970 , with linear interpolation in between. When the tropical ratio is above 0.5 , a linear interpolation is used to assign values between $20^{\circ}$ and $25^{\circ}$ latitude. The more recent tropical values are assigned larger noise-to-signal ratios to account for a possible decrease in quality in recent years as local less developed governments took over the making and keeping of records.

The OI uses these statistics and between one and nine GHCN differences over a $15^{\circ}$ square centered on the $5^{\circ}$ grid box of interest. The OI analyzes the difference for the central box, with damping of the difference when 
TABLE 1. Sum of the OI weights for the given values of noise/ signal variance ratios and one difference at the center (1C), one difference offset by one grid box (1O), and with values filled in all nine grid squares (F9).

\begin{tabular}{cccc}
\hline \hline $\begin{array}{c}\text { Noise-to-signal } \\
\text { variance }\end{array}$ & $1 \mathrm{C}$ & $1 \mathrm{O}$ & F9 \\
\hline 0.5 & 0.67 & 0.25 & 0.91 \\
1.0 & 0.50 & 0.18 & 0.81 \\
\hline
\end{tabular}

there are few differences or when the assigned error is high. Table 1 illustrates the amount of damping for a single observation at the center or offset by one grid square, and when all nine grid squares have defined differences. This analyzed difference is added to the reconstruction to form the gauge-adjusted value. When the sum of the weights indicated in Table 1 approaches 1, the adjusted value approaches the GHCN, while the adjusted value is near the reanalysis when the sum of the weights is low.

The reinjection of gauge data has practically no influence on ocean areas. For land areas it also has only a slight influence on the global spatial mean (Fig. 8). Over land areas the GHCN trend over the analysis period is $1.6 \mathrm{~mm}$ month $^{-1}(100 \mathrm{yr})^{-1}$, while the EOF1 $(\mathrm{GHCN}+\mathrm{CCA})$ with reinjection has a trend of $0.7 \mathrm{~mm} \mathrm{month}{ }^{-1}(100 \mathrm{yr})^{-1}$. Most interannual variations are similar in both, but the GHCN has stronger interannual amplitudes, especially in the more recent decades when sampling is better. Stronger amplitudes may contribute to the stronger GHCN trends compared to the reconstruction.

Reinjection causes greater spatial variations over land areas, as shown by the spatial RMS differences between reconstructions and GHCN (Fig. 9). Without reinjection the spatial RMS difference from GHCN is much larger, as expected. With reinjection there is still a spatial RMS difference from GHCN, because the reinjection is a statistical adjustment and not a simple replacement, but the difference is much less. Reinjection of gauge data improves the land-area analysis for local evaluations and evaluations of seasonal or shorter-period variations that may not be fully resolved by the reconstruction modes. As shown by the land-area averages, the reinjection of data only slightly influences large-scale variations.

\section{e. Summary of method}

The improved reconstruction method has three basic steps. First, an annual average anomaly first guess is reconstructed (outlined on the upper left of Fig. 10). In the figure, arrows show the information flow in this step. Second, the anomaly increment between the annual average guess and the monthly average is reconstructed (outlined on the upper right of Fig. 10). Third, the annual

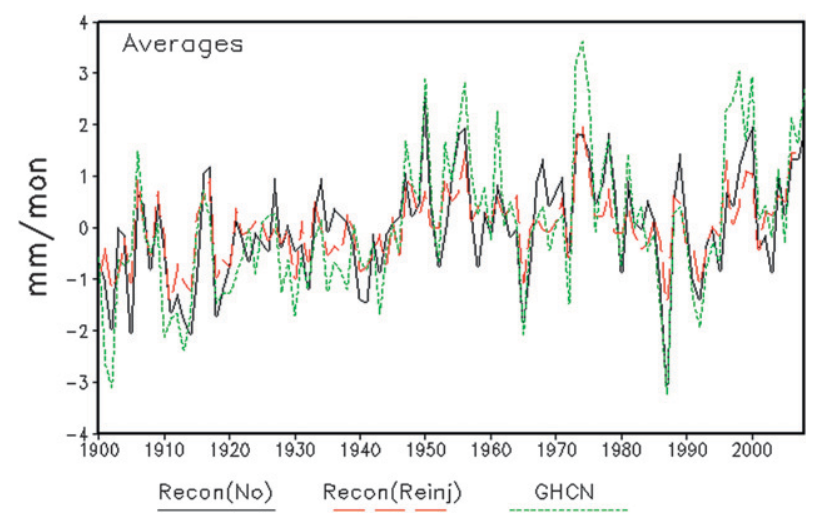

FIG. 8. Global and annual land-area average precipitation anomalies for EOF1(GHCN + CCA) reconstructions without (No) and with (Reinj) GHCN reinjection. The GHCN average is also shown.

guess is added to the monthly increment to form a monthly anomaly reconstruction, which is then further adjusted by statistical reinjection of the gauge data where those data are available (outlined on the lower center of Fig. 10). The anomaly statistics for all of these analyses are based on GPCP.

The annual first-guess analysis is computed by fitting historical data to a set of 10 annual global EOF modes. The EOF modes are based on GPCP anomalies from 1979 to 2008. Two types of annual average historical precipitation anomaly estimates were tested for the first guess: GHCN anomalies alone and GHCN combined with CCA. The annual CCA anomalies are based on correlations with annual SST and SLP anomalies and are used only over oceanic areas where there is no GHCN. Both SST and SLP annual anomalies are based on analyses of those fields, which have been historically sampled over oceanic regions. The SST data used are

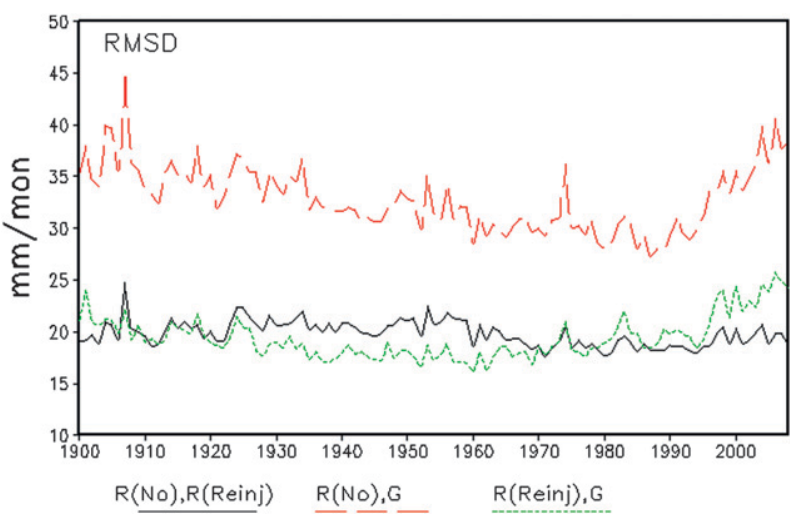

FIG. 9. Global land-area spatial RMS differences between EOF1 $(\mathrm{GHCN}+\mathrm{CCA})$ without (No) and with (Reinj) GHCN reinjection and between each reconstruction and GHCN $(\mathrm{G})$. For clarity annual averages are shown. 


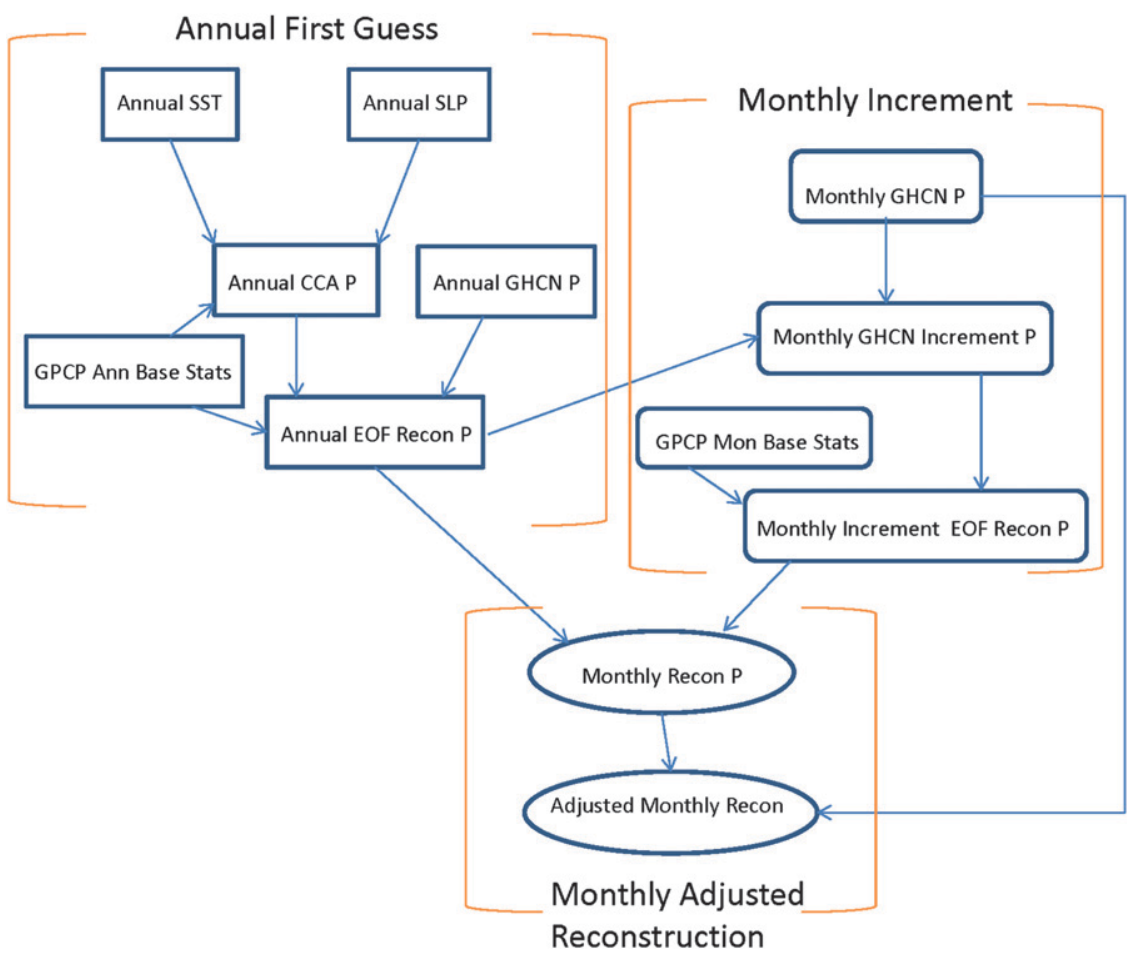

FIG. 10. The processing steps needed to produce the reconstruction.

from the Smith et al. (2008a) reconstruction, which was developed for analysis of climate-scale SST variations. The SLP data are from the Allan and Ansell (2006) reconstruction, which was developed for long-term monitoring of SLP variations. We found that the annual analysis using both GHCN and CCA inputs are able to take advantage of information from the CCA while adjusting the oceanic spatial patterns of variations to better match those of the base data, compared to the raw CCA patterns.

The monthly increment analysis uses monthly GHCN anomaly increments from the annual average. The CCA is not used for the monthly increment analysis because it is less reliable for shorter time periods and because the GHCN sampling is generally sufficient to resolve monthto-month variations. The monthly increments are added to the first-guess annual analysis to give a monthly anomaly analysis. The GHCN monthly gauge anomalies are then locally reinjected into the monthly anomaly analysis using optimum interpolation to minimize errors relative to the gauges where they are available.

\section{f. Spatial scales of variations}

The spatial scales of precipitation variations over the reconstruction period are illustrated by the difference between the 1979 and 2008 climatology, shown in Fig. 1, and the reconstruction 1909-38 climatology (Fig. 11). Most of the differences have scales of several thousand kilometers or larger. Over the reconstruction period the tropical precipitation generally increased, although there are regions in the tropics where precipitation decreased. The generally stronger convection near the equator appears to be associated with stronger subsidence in the poleward branches of the Hadley cells, leading to drying in some of the dry-zone regions. That may be responsible for the boreal winter drying along western North America and in the Mediterranean region. The differences also suggest zonal shifts in the most intense tropical precipitation, with more intense Pacific precipitation shifting eastward. Zonal shifts influence the strength of the zonal tropical circulation cells, such as the Walker cell, which could be responsible for the alternating positive and negative differences around the equator. The zonal shifts also greatly influence precipitation teleconnections to higher latitudes, which could be affecting the changes in JJA monsoon precipitation across southern Asia and the JJA increase in central North America.

\section{Discussion}

We show a method for improved statistical reconstruction of ocean-area historical monthly precipitation. Improvements include using global modes to eliminate seams in the global analysis, computation of a first guess using a low number of modes and increment 


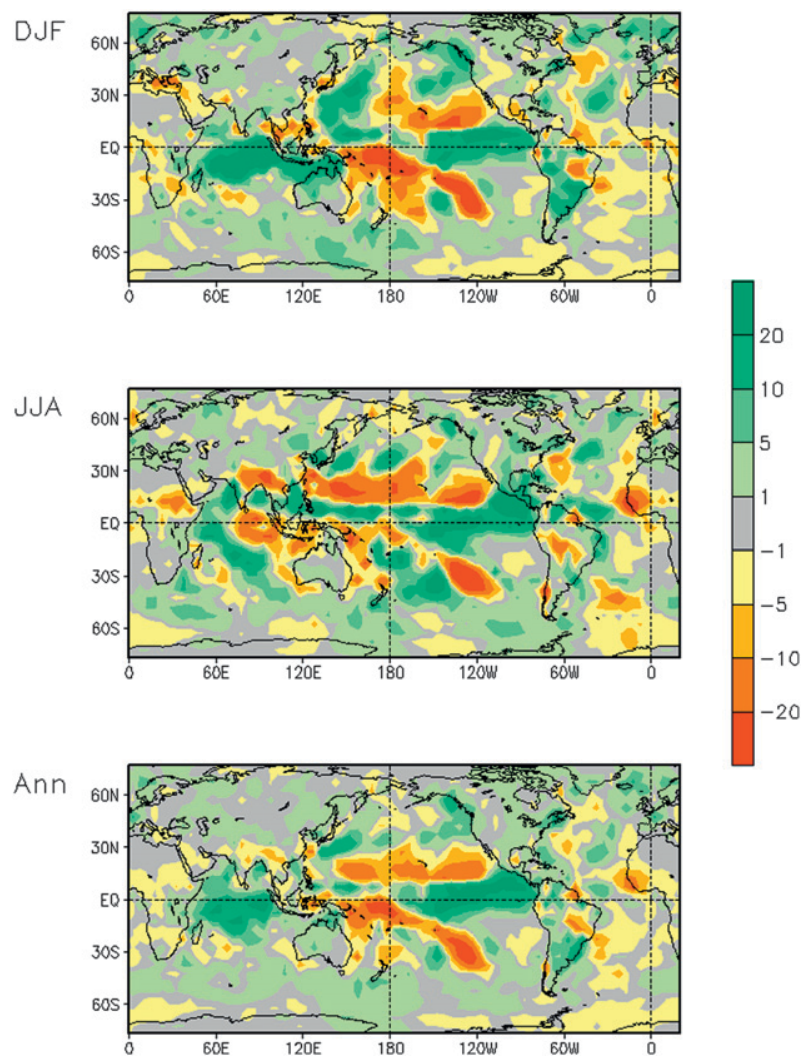

FIG. 11. Difference between the 1979-2008 climatology and the 1909-38 climatology for DJF, JJA, and all months.

reconstructions to adjust the guess, and statistical optimal reinjection of gauge data where they are available. The improvement that makes the largest difference for multidecadal variations is the computation of the annual first guess using both gauge data and oceanic data that are correlated with precipitation. The SST and SLP are included in the first guess through a CCA process. Those correlated data give similar interannual variations of annual averages, but they strengthen the multidecadal variations since 1900.

Compared to earlier studies, this method yields similar large-scale variations but with better resolution of spatial patterns. The reinjection of gauge data also improves resolution of smaller-scale features in the land-area spatial patterns. This makes the improved reconstruction more appropriate for regional studies of oceanic and land-ocean precipitation variations.

This study of oceanic precipitation variations is ongoing. An important aspect of this to be documented in a future report is error estimates for the reconstruction. Some components of the error can be quantified using methods already developed, such as CCA errors discussed by Shen et al. (2001), EOF reconstruction errors in the modes discussed by Shen et al. (2004), and a method for computing the error of individual GHCN grid squares given by Shen et al. (2007). Other error components include error from using a limited set of modes, bias errors, and changes in errors from the reinjection of gauge data. All of these errors need to be estimated and their influence on this multistep reconstruction requires quantification. Because errors of averages are often desired, methods are needed for computing the errors for both monthly maps and temporal and spatial averages.

The error component that may be most difficult to estimate is the bias error. Bias is also the error component with greatest influence on multidecadal variations, and therefore understanding the bias is important. Comparison of the ocean-area reconstruction without CCA (Fig. 4) and with CCA (Fig. 6) shows the systematic difference in interannual variations that occurs from the inclusion of oceanic data through the CCA. That difference implies a large-scale bias uncertainty, either from bias in the data or from systematic underestimation of multidecadal variations when only gauge data are available. Testing is needed to understand and quantify this bias uncertainty, and to more clearly show which of the two oceanic estimates is most reliable. We believe that including oceanic data through the CCA improves the analysis, but more testing is needed to prove that. Preliminary error analyses indicate that although including CCA slightly increases the random error and also adds significantly to the bias error, the reduction in sampling error if greater, so the reconstruction with CCA included has lower overall error. Documentation of the error of this improved reconstruction is in preparation. The analysis is available online (http://cics. umd.edu/ tsmith/recpr/eof1/full/).

Acknowledgments. This research was supported in part by the National Science Foundation (NSF) Grant AGS-1015926. Shen was also supported by National Science Foundation (NSF) Grant AGS-1015957, a U.S. National Oceanographic and Atmospheric Administration (NOAA) Grant EL133E09SE4048, and a U.S. Department of Energy (DOE) Grant DE-SC0002763. This research was also supported in part by the Cooperative Institute for Climate and Satellites. We thank three anonymous reviewers for their many helpful suggestions and encouragement. The contents of this paper are solely the opinions of the authors and do not constitute a statement of policy, decision, or position on behalf of NOAA or the U.S. Government.

\section{REFERENCES}

Adler, R. F., and Coauthors, 2003: The version-2 Global Precipitation Climatology Project (GPCP) monthly precipitation analysis (1979-present). J. Hydrometeor., 4, 1147-1167. 
Allan, R. J., and T. J. Ansell, 2006: A new globally complete monthly historical gridded mean sea level pressure dataset (HadSLP2): 1850-2004. J. Climate, 19, 5816-5842.

Allan, R. P., and B. J. Soden, 2008: Atmospheric warming and the amplification of precipitation extremes. Science, 321, 1481-1484.

Barnett, T. P., and R. Preisendorfer, 1987: Origins and levels of monthly and seasonal forecast skill for United States surface air temperatures determined by canonical correlation analysis. Mon. Wea. Rev., 115, 1825-1850.

Compo, G. P., J. S. Whitaker, and P. D. Sardeshmukh, 2006: Feasibility of a 100 -year reanalysis using only surface pressure data. Bull. Amer. Meteor. Soc., 87, 175-190.

Durack, P. J., S. E. Wijffels, and R. J. Matear, 2012: Ocean salinities reveal strong global water cycle intensification during 1950 to 2000. Science, 336, 455-458.

Efthymiadis, D., M. New, and R. Washington, 2005: On the reconstruction of seasonal oceanic precipitation in the presatellite era. J. Geophys. Res., 110, D06103, doi:10.1029/ 2004JD005339.

Held, I. M., and B. J. Soden, 2006: Robust responses of the hydrological cycle to global warming. J. Climate, 19, 5686-5699.

Hsu, K.-L., X. Gao, S. Sorooshian, and H. V. Gupta, 1997: Precipitation estimation from remotely sensed information using artificial neural networks. J. Appl. Meteor., 36, 1176-1190.

Huffman, G. J., and Coauthors, 1997a: The Global Precipitation Climatology Project (GPCP) combined precipitation datasets. Bull. Amer. Meteor. Soc., 78, 5-20.

_ - and Coauthors, 1997b: The TRMM Multisatellite Precipitation Analysis (TMPA): Quasi-global, multiyear, combined-sensor precipitation estimates at fine scales. J. Hydrometeor., 8, 38-55.

— , R. F. Adler, D. T. Bolvin, and G. Gu, 2009: Improving the global precipitation record: GPCP version 2.1. Geophys. Res. Lett., 36, L17808, doi:10.1029/2009GL040000.

Hulme, M., T. J. Osborn, and T. C. Johns, 1998: Precipitation sensitivity to global warming: Comparison of observations with HadCM2 simulations. Geophys. Res. Lett., 25, 3379-3382.

Joyce, R. J., J. E. Janowiak, P. A. Arkin, and P. Xie, 2004: CMORPH: A method that produces global precipitation estimates from passive microwave and infrared data at high spatial and temporal resolution. J. Hydrometeor., 5, 487-503.

Ren, L., and S. C. Riser, 2009: Seasonal salt budget in the northeast Pacific Ocean. J. Geophys. Res., 114, C12004, doi:10.1029/ 2009JC005307.

Rudolf, B., 2005: Global precipitation analysis products of the GPCC. Climate Monitoring_Tornadoklimatologie-Aktuelle Ergebnisse des Klimamonitorings, Klimastatusbericht Series 2004, DWD, 163-170.

Schneider, U., T. Fuchs, A. Meyer-Christoffer and B. Rudolf, 2008: Global precipitation analysis products of the GPCC. Global Precipitation Climatology Centre, DWD, 12 pp. [Available online at http://www.tam.cec.org/Atlas/Files/Precipitation/ GPCC_intro_products_2008.pdf.]

Shen, S. S. P., W. K. M. Lau, K.-M. Kim, and G. Li, 2001: A canonical ensemble correlation prediction model for seasonal precipitation anomaly. NASA/TM-2001-209989, 68 pp. [Available online at http://ntrs.nasa.gov/archive/nasa/casi.ntrs.nasa gov/20010102849_2001168967.pdf.]

— , A. N. Basist, G. Li, C. Williams, and T. R. Karl, 2004: Prediction of sea surface temperature from the global historical climatology network data. Environmetrics, 15, 233-249.

,- H. Yin, and T. M. Smith, 2007: An estimate of the sampling error variance of the gridded GHCN monthly surface air temperature data. J. Climate, 20, 2321-2331.
Smith, T. M., R. W. Reynolds, R. E. Livezey, and D. C. Stokes, 1996: Reconstruction of historical sea surface temperatures using empirical orthogonal functions. J. Climate, 9, 1403-1420.

, R. E. Livezey, and S. S. P. Shen, 1998: An improved method for analyzing sparse and irregularly distributed SST data on a regular grid: The tropical Pacific Ocean. J. Climate, 11, 1717-1729.

— , R. W. Reynolds, T. C. Peterson, and J. Lawrimore, 2008a: Improvements to NOAA's historical merged land-ocean surface temperature analysis (1880-2006). J. Climate, 21, 2283-2296.

—, M. R. P. Sapiano, and P. A. Arkin, 2008b: Historical reconstruction of monthly oceanic precipitation (1900-2006). J. Geophys. Res., 113, D17115, doi:10.1029/2008JD009851.

, P. A. Arkin, and M. R. P. Sapiano, 2009a: Reconstruction of near-global annual precipitation using correlations with sea surface temperature and sea level pressure. J. Geophys. Res., 114, D12107, doi:10.1029/2008JD011580.

M. R. P. Sapiano, and P. A. Arkin, 2009b: Modes of multidecadal oceanic precipitation variations from a reconstruction and AR4 model output for the 20th century. Geophys. Res. Lett., 36, L14708, doi:10.1029/2009GL039234.

P. A. Arkin, M. R. P. Sapiano, and C.-Y. Chang, 2010: Merged statistical analyses of historical monthly precipitation anomalies beginning 1900. J. Climate, 23, 5755-5770.

Sorooshian, S., K.-L. Hsu, X. Gao, H. V. Gupta, B. Imam, and D. Braithwaite, 2000: Evaluation of PERSIANN system satellitebased estimates of tropical rainfall. Bull. Amer. Meteor. Soc., 81, 2035-2046.

Trenberth, K. E., and J. W. Hurrell, 1994: Decadal atmosphericocean variations in the Pacific. Climate Dyn., 9, 303-319, doi:10.1007/BF00204745.

—_ and Coauthors, 2007: Observations: Surface and atmospheric climate change. Climate Change 2007: The Physical Science Basis, S. Solomon et al., Eds., Cambridge University Press, 235-336.

Vose, R. S., T. C. Peterson, and M. Hulme, 1998: The Global Historical Climatology Network Precipitation Database: Version 2.0. Proc. Ninth Symp. on Global Change Studies, Phoenix, AZ, Amer. Meteor. Soc., 103-105.

Woodruff, S. D., H. F. Diaz, J. D. Elms, and S. J. Worley, 1998: COADS release 2 data and metadata enhancements for improvements of marine surface flux fields. Phys. Chem. Earth, 23, 517-527.

Xie, P., and P. A. Arkin, 1996: Global monthly precipitation estimates from satellite-observed outgoing longwave radiation. J. Climate, 11, 137-164.

— monthly analysis based on gauge observations, satellite estimates and numerical model outputs. Bull. Amer. Meteor. Soc., 78, 2539-2558.

, M. Chen, J. E. Janowiak, P. A. Arkin, and T. M. Smith, 2001: Reconstruction of the oceanic precipitation: Preliminary results. Proc. 26th Climate Diagnostics and Prediction Workshop, La Jolla, CA, NOAA. [Available online at http://www. cpc.ncep.noaa.gov/products/outreach/proceedings/cdw26_ proceedings/xie.pdf.]

Yaremchuk, M., 2006: Sea surface salinity constrains rainfall estimates over tropical oceans. Geophys. Res. Lett., 33, L15605, doi:10.1029/2006GL026582.

- Z. Z. Yu, and J. McCreary, 2005: River discharge into the Bay of Bengal in an inverse ocean model. Geophys. Res. Lett., 32, L16605, doi:10.1029/2005GL023750.

Yu, L., 2011: A global relationship between the ocean water cycle and near-surface salinity. J. Geophys. Res., 116, C10025, doi:10.1029/2010JC006937. 